\title{
Interventional trials with anticoagulants in acutely ill medical patients: a methodological pitfall?
}

\author{
Francesco Violi • Ludovica Perri • Lorenzo Loffredo
}

Received: 16 May 2013/ Accepted: 5 June 2013/Published online: 16 June 2013

(c) SIMI 2013

Autopsy and retrospective studies [1-4] lead to believe that patients hospitalized for acute medical illness are at higher risk of deep venous thrombosis (DVT) and pulmonary embolism (PE) and that anticoagulant treatment was, therefore, necessary to prevent such vascular outcomes. Interventional trials consistently showed that prophylaxis with anticoagulants reduces the risk of composite endpoints of DVT, PE and DVT-related death in patients hospitalized for acute medical illness while no effect has been detected for total mortality [5]. These results prompted to recommend the use of anticoagulant prophylaxis in patients hospitalized for acute medical illness [6], but despite this, there is a large underuse of anticoagulant prophylaxis in the medical wards of hospitals [7, 8].

The scarce use of anticoagulants in acutely ill medical is almost evident in a multicenter clinical registry (The REPOSI study) performed by investigating patients hospitalized in Italian divisions of internal medicine [9].

Thus, Marcucci et al. [9] included 1,121 acutely ill medical patients and followed them up during the hospital stay and until 3 months after hospital discharge. Patients included were very old, with a mean age of 82 years; males and females were equally distributed. Among 1,121 patients, $171(15.2 \%)$ were treated with thromboprophylaxis (TP) including low molecular weight heparin $(n=158)$, unfractionated heparin $(n=4)$ and fondaparinux $(n=9)$. Administration of anticoagulants was

This comment refers to the article available at doi:10.1007/s11739-013-0944-8.

F. Violi $(\bowtie) \cdot$ L. Perri · L. Loffredo

I Clinica Medica, Sapienza University of Rome,

Viale del Policlinico 155, 00161 Rome, Italy

e-mail: francesco.violi@uniroma1.it unrelated to the current guidelines of this setting as only $14.9 \%$ of treated patients met the criteria of ACCP 2004 guidelines [10]. Among the putative risk factors considered, impaired global performance, as assessed by Barthel index [11], length of hospital stay and acute respiratory failure were independent predictors of TP during the hospitalization.

Because the rate of patients treated with TP was low, the study offers an interesting chance to appreciate the rate of DVT in a relatively large population of acutely ill medical patients. Figure 1 depicts the rate of DVT in patients treated or not with TP. During the hospital stay among 950 untreated patients, DVT was diagnosed in $5(0.5 \%)$ while $1(0.6 \%)$ DVT was observed in the 171 treated patients; in the 3-month follow-up only three untreated and one treated patients experienced DVT. Taking into account the entire follow-up, $8(0.8 \%)$ untreated and $2(1.1)$ treated patients experienced DVT. Pulmonary embolism was detected in two patients with DVT (one treated and one untreated) only after hospital discharge.

We have recently reviewed the incidence of DVT in the interventional trials with anticoagulants performed in acutely ill medical patients [12].

The rate of DVT was quite different if symptomatic or asymptomatic events were separately considered [12]. Thus, asymptomatic DVT was much more frequent compared to symptomatic ones with a large variation ranging as high as $28 \%$ to as low as $2.3 \%$ with an average of $4.7 \%$ [12]. The rate of symptomatic DVT was much less with values from as high as $1.5 \%$ to as low as $0.8 \%$ and an average of $0.99 \%$ [12]. Despite Marcucci et al. [9] reported the incidence of only symptomatic DVT, we believe that these data add important news on this topic. Thus, they found that during the hospital stay the rate of symptomatic DVT was a little bit less $(0.5 \%)$ compared to 


\section{DVT in REPOSI}

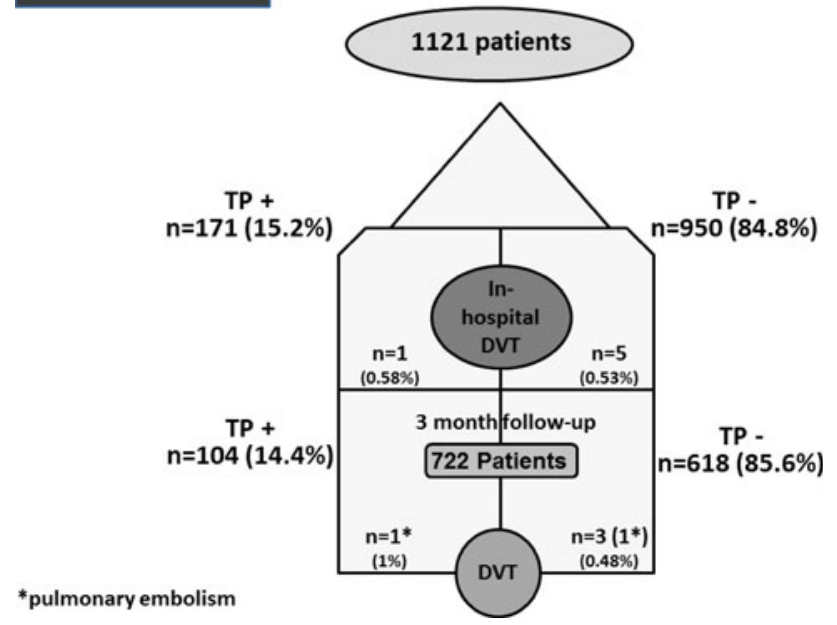

Fig. 1 DVT in the REPOSI study

the rate observed in the interventional trials. This is particularly intriguing taking into account that the REPOSI study [9] included patients much older than those enrolled in the interventional trials with anticoagulants [12]. This would suggest that in the real world of acutely ill medical patients the rate of symptomatic DVT is relatively low even in case of very old people. It is likely that also in the study by Marcucci et al. [9] asymptomatic DVT was not diagnosed because this was out of the scope of the study. However, the clinical impact of asymptomatic DVT could not be clinically relevant as suggested by the absence of pulmonary embolism during the intra-hospital stay. How these findings fit with the interventional trials and the guidelines on this topic suggesting the potential relevance of anticoagulation therapy in acutely ill medical patients? To investigate this issue we reviewed the scientific background which prompted to plan interventional trials with anticoagulants. The studies were essentially necropsy analysis of patients hospitalized in internal medicine wards, who died from pulmonary embolism [1-4]. In a large study performed in different clinical settings it appeared that patients hospitalized in the department of internal medicine had an even higher risk of developing pulmonary embolism compared to other departments including that of general surgery, infectious disease, oncology and orthopaedics [1]. Apart from the retrospective nature of these analyses, it should be underscored that none of these studies provided clinical details of the medical illness which predisposed to venous thromboembolism; in rare cases a list of concomitant medical diseases was reported. It is, therefore, very difficult to appreciate if DVT and pulmonary embolism were a common feature of medical patients or if specific clinical illness could predispose to venous thrombosis. Despite these serious limitations and, overall, the lack of prospective studies which validated the relationship between acutely ill medical patients and the risk of venous thrombosis, several trials have been planned to assess the clinical efficacy on anticoagulants in this setting. The inclusion criteria of the Medenox [13], the landmark of these clinical trials, were the presence of heart failure, infections, acute respiratory disease, rheumatic arthritis and episodes of inflammatory bowel disease; age $>75$, cancer, previous venous thrombosis and obesity were considered additional risk factors. Similar inclusion criteria were adopted by following trials [14-17]. We found that in these trials the majority of acutely ill medical patients (about $80 \%$ ) was affected by heart failure, infections or acute respiratory disease; of note, none of these diseases has been shown to be associated with a higher risk of venous thrombosis with the exception of a retrospective analysis showing a significant association between infections and venous thrombosis [11]. Furthermore, no clinical details of the acute medical illness were requested. For instance, we did not find a clear definition of acute respiratory disease, which is crucial in this setting as the severity of lung disease may be a key risk factor for mortality [12].

Similar critical issues can be found in the last trial, the Magellan trial [18], which compared $40 \mathrm{mg}$ once daily enoxaparin versus $10 \mathrm{mg}$ once daily rivaroxaban, an inhibitor of $\mathrm{Xa}$, in 8,101 patients hospitalized for acute medical illness. Also in this trial the majority of patients were affected by infectious disease, heart failure and respiratory insufficiency. A high number $(17 \%)$ of patients suffered from acute ischemic stroke, which is relatively unusual in a section of internal medicine as also detected by the Marcucci's study [9], which showed a prevalence of ischemic stroke $<3 \%$. Apart from heart failure, which was actually defined, also the Magellan failed to deeper specify the clinical characteristics and severity of diseases such as infections and respiratory insufficiency at admission. Finally, the authors did not provide information on whether a specific clinical pathology carried a higher risk of venous thromboembolism.

Based on this consideration, should clinicians treat patients hospitalized for acutely ill medical or use TP only in patients who are at higher risk of venous thrombosis? In the last guidelines [5], on this topic the authors suggest the use of the PADUA score [19] to identify patient candidates for TP. However, the PADUA score is a result of a retrospective study, which, in our opinion, should be prospectively validated before being considered as a diagnostic tool to implement TP. A typical case is age $>75$, which is indicated as a risk factor in the current ACCP guidelines [5] and in the PADUA score while the study by Marcucci et al. [9] seems to deny it as the rate of venous thrombosis was relatively low even in a population much older than 75. Also, the cost of TP in such population cannot be 
Bleeding

complication

in REPOSI
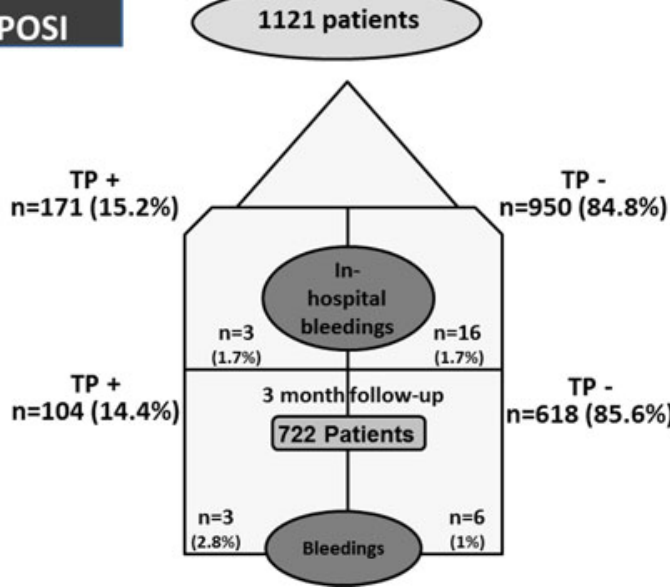

Fig. 2 Bleeding complication (major and minor) in the REPOSI study

overlooked as TP was associated with a higher risk of bleeding (major plus minor) in the intra-hospital period as well as after discharge. As shown in the Fig. 2, taking into account the entire follow-up the rate of bleeding was $3.5 \%$ in treated and $2.7 \%$ in untreated patients.

The analysis of these findings leads us to suggest that a more appropriate methodological approach should be followed to investigate the occurrence of venous thrombosis and eventually PE in acutely ill medical patients. The first objective would be to clearly define the rate of DVT and $\mathrm{PE}$ in a unselected population hospitalized in internal medicine departments; to this purpose an international registry should be mandatory also to evidence if differences among races do exist. The second objective would be the identification of clinical settings which are candidates to TP; this should be achieved by accurate analysis of baseline clinical characteristics, severity of the disease and existence of co-morbidities. The results of these findings could be a prerequisite to plan interventional trials with anticoagulants in acutely ill medical patients really at risk of DVT.

In conclusion, in the real world of internal medicine wards described by Marcucci et al. [9], the rate of venous thrombosis in acutely ill medical patients seems to be lower than that previously reported in interventional clinical trials. This is particularly impressive taking into account that most patients were older than 75 years. Use of anticoagulants was low and associated with an increased risk of bleeding; of note, few patients on anticoagulants met the criteria of the ACCP 2004 guidelines [10]. It cannot be excluded that anticoagulant's under-prescription may be dependent on a scarce perception that this setting is actually associated with a high risk of venous thrombosis- related death. This may depend on several factors: (1) lack of prospective studies, which clarify the relationship between DVT and acute medical diseases, (2) no clear definition of medical patients at risk of DVT.

Therefore, an international survey which analyzes the rate of thromboembolism in acutely ill medical patients and the clinical characteristics which predispose to venous thrombosis and develop a risk score is necessary. In the meantime, it could be interesting to establish if some categories of acutely ill medical patients are really at risk of DVT during the intra-hospital stay and may benefit from TP.

Conflict of interest None.

\section{References}

1. Lindblad B, Sternby NH, Bergqvist D (1991) Incidence of venous thromboembolism verified by necropsy over 30 years. BMJ 302:709-711

2. Sandler DA, Martin JF (1989) Autopsy proven pulmonary embolism in hospital patients: are we detecting enough deep vein thrombosis? J R Soc Med 82:203-205

3. Rubinstein I, Murray D, Hoffstein V (1988) Fatal pulmonary emboli in hospitalized patients. An autopsy study. Arch Intern Med 148:1425-1426

4. Stein PD, Henry JW (1995) Prevalence of acute pulmonary embolism among patients in a general hospital and at autopsy. Chest 108:978-981

5. Kahn SR, Lim W, Dunn AS, Cushman M, Dentali F, Akl EA, Cook DJ, Balekian AA, Klein RC, Le H, Schulman S, Murad MH (2012) Prevention of VTE in nonsurgical patients: antithrombotic therapy and prevention of thrombosis, 9th edn: American college of chest physicians evidence-based clinical practice guidelines. Chest 141:e195S-e226S

6. Geerts WH, Bergqvist D, Pineo GF, Heit JA, Samama CM, Lassen MR, Colwell CW (2008) Prevention of venous thromboembolism: American college of chest physicians evidencebased clinical practice guidelines (8th edn). Chest 133:381S$453 \mathrm{~S}$

7. Wittkowsky AK (2004) Effective anticoagulation therapy: defining the gap between clinical studies and clinical practice. Am J Manag Care 10:S297-306; discussion S312-297

8. Ageno W (2012) Do medical patients need to receive pharmacologic prophylaxis for the prevention of venous thromboembolism? Intern Emerg Med 7(Suppl 3):S189-S192

9. Marcucci M, Iorio A, Nobili A, Tettamanti M, Pasina L, Djade CD, Marengoni A, Salerno F, Corrao S, Mannucci PM, REPOSI (REgistro POliterapie Società Italiana di Medicina Interna) Investigators (2013) Prophylaxis of venous thromboembolism in elderly patients with multimorbidity. Intern Emerg Med. doi: 10.1007/s11739-013-0944-8

10. Geerts WH, Pineo GF, Heit JA, Bergqvist D, Lassen MR, Colwell CW, Ray JG (2004) Prevention of venous thromboembolism: the seventh ACCP conference on antithrombotic and thrombolytic therapy. Chest 126:338S-400S

11. Shah S, Vanclay F, Cooper B (1989) Improving the sensitivity of the Barthel index for stroke rehabilitation. J Clin Epidemiol 42:703-709

12. Violi F, Perri L, Loffredo L (2013) Should all acutely ill medical patients be treated with antithrombotic drugs? A review of the interventional trials. Thromb Haemost 109:589-595 
13. Samama MM, Cohen AT, Darmon JY, Desjardins L, Eldor A, Janbon C, Leizorovicz A, Nguyen H, Olsson CG, Turpie AG, Weisslinger $N$ (1999) A comparison of enoxaparin with placebo for the prevention of venous thromboembolism in acutely ill medical patients. Prophylaxis in medical patients with enoxaparin study group. N Engl J Med 341:793-800

14. Leizorovicz A, Cohen AT, Turpie AG, Olsson CG, Vaitkus PT, Goldhaber SZ (2004) Randomized, placebo-controlled trial of dalteparin for the prevention of venous thromboembolism in acutely ill medical patients. Circulation 110:874-879

15. Kakkar AK, Cimminiello C, Goldhaber SZ, Parakh R, Wang C, Bergmann JF (2011) Low-molecular-weight heparin and mortality in acutely ill medical patients. N Engl J Med 365: 2463-2472

16. Hull RD, Schellong SM, Tapson VF, Monreal M, Samama MM, Nicol P, Vicaut E, Turpie AG, Yusen RD (2010) Extendedduration venous thromboembolism prophylaxis in acutely ill medical patients with recently reduced mobility: a randomized trial. Ann Intern Med 153:8-18

17. Cohen AT, Davidson BL, Gallus AS, Lassen MR, Prins MH, Tomkowski W, Turpie AG, Egberts JF, Lensing AW (2006) Efficacy and safety of fondaparinux for the prevention of venous thromboembolism in older acute medical patients: randomised placebo controlled trial. BMJ 332:325-329

18. Cohen AT, Spiro TE, Buller HR, Haskell L, Hu D, Hull R, Mebazaa A, Merli G, Schellong S, Spyropoulos AC, Tapson V (2013) Rivaroxaban for thromboprophylaxis in acutely ill medical patients. N Engl J Med 368:513-523

19. Barbar S, Noventa F, Rossetto V, Ferrari A, Brandolin B, Perlati M, De Bon E, Tormene D, Pagnan A, Prandoni P (2010) A risk assessment model for the identification of hospitalized medical patients at risk for venous thromboembolism: the Padua Prediction Score. J Thromb Haemost 8:2450-2457 